\title{
GRAINE project: An overview and status of the 2015 balloon-borne experiment with emulsion gamma-ray telescope
}

\author{
Satoru Takahashi* \\ Kobe University, Nada, Kobe 657-8501, Japan \\ E-mail: satorueradix.h.kobe-u.ac.jp \\ for the GRAINE collaboration \\ Aichi University of education, ISAS/JAXA, Kobe University, Nagoya University, Okayama \\ University of science, Utsunomiya University
}

\begin{abstract}
We are furthering GRAINE project, $10 \mathrm{MeV}-100 \mathrm{GeV}$ cosmic gamma-ray observation with a precise (0.08deg@1-2GeV) and polarization sensitive large aperture area $\left(\sim 10 \mathrm{~m}^{2}\right)$ emulsion telescope by repeated long duration balloon flights. In 2015, we performed a balloon experiment on Japan-Australia JAXA scientific ballooning at Balloon Launching Station Alice Springs with a $3780 \mathrm{~cm}^{2}$ aperture area and 14.4 hour flight duration. In this experiment, we aim to detect a wellknown bright gamma-ray source, Vela pulsar, and to demonstrate the overall performance of the emulsion gamma-ray telescope. And we start the observation with the highest imaging resolution and polarization sensitivity. And phase resolving of the pulse emission from the Vela pulsar is also challenged.
\end{abstract}

The 34th International Cosmic Ray Conference,

30 July- 6 August, 2015

The Hague, The Netherlands

${ }^{*}$ Speaker. 


\section{Introduction}

The observation of high-energy cosmic gamma-rays provides us with direct information of high-energy phenomena in the universe. Currently, AGILE and Fermi-LAT are observing gammaray sky and many understandings are being brought to us. However, past and current observations have significant limitations. The improvement of angular resolution and polarization sensitivity is one of keys for a breakthrough of the limitations.

We are furthering GRAINE project, $10 \mathrm{MeV}-100 \mathrm{GeV}$ cosmic gamma-ray observation with a precise (0.08deg@1-2GeV) and polarization sensitive large aperture area $\left(\sim 10 \mathrm{~m}^{2}\right)$ emulsion telescope by repeated long duration balloon flights [1].

We demonstrated the feasibility and performance of the emulsion gamma-ray telescope using accelerator beams with gamma-rays/electrons/muons and atmospheric gamma-rays at mountain height.

In 2011, the first balloon-borne, emulsion gamma-ray telescope experiment was successfully performed with a $125 \mathrm{~cm}^{2}$ aperture area and 4.3 hour flight duration. By the flight data analysis, we systematically detected gamma-ray events down to $<50 \mathrm{MeV}$ gamma-ray energy, up to $>45$ degree incident zenith angle and above $97 \%$ detection reliability. And we created a calibration method for gamma-ray data using a flight data and demonstrated a high angular resolution (1.0 degree around $100 \mathrm{MeV}$ ) and a sufficient timing resolution ( 0.21 second) using the flight data. Timestamped gamma-ray events and combined attitude data, a determination of gamma-ray arrival direction on celestial coordinates was performed and we established this proceedure in this experiment. And the first understanding of the background was obtained with a balloon flight of the emulsion gammaray telescope. [3, 4]

In 2015, we performed a balloon experiment on Japan-Australia JAXA scientific ballooning at Balloon Launching Station (BLS) Alice Springs with a $3780 \mathrm{~cm}^{2}$ aperture area and 14.4 hour flight duration. In this experiment, we aim to detect a well-known bright gamma-ray source, Vela pulsar, and to demonstrate the overall performance of the emulsion gamma-ray telescope. And we start the observation with the highest imaging resolution and polarization sensitivity. And phase resolving of the pulse emission from the Vela pulsar is also challenged. The design, improvements, preparations for the 2015 balloon experiment and its experiment are described in this paper.

\section{Design, improvements and preparations for the balloon experiment}

Based on the experiences and achievements in the 2011 balloon experiment, we designed the balloon experiment. Vela pulsar move with 22 degree minimum zenith at 17:39 Australian Central Standard Time (ACST) on 15th May 2015 at Alice Springs, and within 45 degree zenith (roughly corresponding to a field of view of the emulsion gamma-ray telescope) for 6.5 hours (14:24 - 20:54). We calculated a detection significance of Vela pulsar with the emulsion gammaray telescope. With $2500 \mathrm{~cm}^{2}$ aperture area $\left(125 \mathrm{~cm}^{2}(3.5 \mathrm{~cm}\right.$ thickness $) \times 20$ units $)$ and 6.5 hour exposure at $5 \mathrm{~g} / \mathrm{cm}^{2}$ atmospheric depth, Vela pulsar can be detected significantly.

Various improvements were performed based on the experiences and achievements in the 2011 balloon experiment. 
In 2011 balloon experiment, we partially used self-produced emulsion gel films. Then we obtained high track finding efficiency in a single film (97.7\%) and uniform track finding efficiency for each incident zenith angle ( $0-54.5$ degree). In the next balloon experiment, we fully use self-produced emulsion gel films. By using self-produced emulsion gel film, high and uniform track finding efficiency and high signal - noise ratio can be performed. As a result, an efficient analysis work, reduction of processing load, high reduction power of background tracks, high detection efficiency of gamma-ray events and low energy threshold can be achieved. We produced emulsion gel in January 2014 - June 2014 and October 2014 - February 2015. The emulsion gel, $166.4 \mathrm{~kg}$, was produced with $55 \%$ occupancy of silver bromide cristals in a volume for the converter (, flatness compensation films and unit alignment films) in order to make high conversion efficiency and make thin a total thickness. The emulsion gel, $28.2 \mathrm{~kg}$, was produced with $44 \%$ occupancy of silver bromide cristals in a volume for the timestamper and calorimeter in order to minimize thickness in $\mathrm{g} / \mathrm{cm}^{2}$ keeping track finding efficiency. Total amount of produced emulsion gel was $194.7 \mathrm{~kg}$ with 245 productions. This is the first emulsionic experiment that such a amount of emulsion gel has been produced by ourselves. The emulsion gel was poured in May - June 2014 and December 2014 - March 2015. The $55 \%$ occupied emulsion gel film has $320 \mu \mathrm{m}$ thickness, consisting of $70 \mu \mathrm{m}$ thick emulsion layers coated on both sides of a $180 \mu \mathrm{m}$ thick polystyrene (PS) base for the converter (, flatness compensation films and unit alignment films). The $44 \%$ occupied emulsion gel film has $264 \mu \mathrm{m}$ thickness, consisting of $42 \mu \mathrm{m}$ thick emulsion layers coated on both sides of a $180 \mu \mathrm{m}$ thick PS base for the timestamper, has $120 \mu \mathrm{m}$ thickness, consisting of $40 \mu \mathrm{m}$ thick emulsion layers coated on both sides of a $40 \mu \mathrm{m}$ thick PS base for the narrow part of the timestamper, and has $270 \mu \mathrm{m}$ thickness, consisting of $45 \mu \mathrm{m}$ thick emulsion layers coated on both sides of a $180 \mu \mathrm{m}$ thick PS base for the calorimeter. Total amount of poured gel was $66.83 \mathrm{~m}^{2}$ with $143.9 \mathrm{~kg}$ gel. More details of emulsion film production are described in Ref. [5].

We use an emulsion layer as a main gamma-ray target without a metal foil (by tuned thickness and density of emulsion layer and tuned a number of stacked emulsion films in order to keep total conversion efficiency). Hereby, it can make fully visible around a conversion point. Very precise measurement suppressed multiple coulomb scattering can be performed by grain-by-grain measurement just beneath a conversion point. As a result, higher angular resolution for gamma-rays (0.63deg @ $100 \mathrm{MeV}$ ) and higher sensitivity to gamma-ray polarization can be achieved. And it can make robust to slipping between emulsion films due to a stack without a material with different thermal expansion coefficient.

We enlarged an unit size of the emulsion film stack from $12.5 \times 10.0 \mathrm{~cm}^{2}(\times 100 \mathrm{films}$, converter ( $3.5 \mathrm{~cm}$ thickness in the 2011 balloon experiment)) to $37.8 \times 25.0 \mathrm{~cm}^{2}(\times 100 \mathrm{films}$, converter (3.2 cm thickness)). A loss ratio due to an edge effect was improved with a factor $\sim 3$. Total aperture area was also enlarged from $2500 \mathrm{~cm}^{2}\left(12.5 \times 10.0 \mathrm{~cm}^{2} \times 20\right.$ units $)$ to $3780 \mathrm{~cm}^{2}$ $\left(37.8 \times 25.0 \mathrm{~cm}^{2} \times 4\right.$ units). Reducing total number of emulsion films (from 20 units to 4 units) allows us to reduce handling load of emulsion films.

Multi-stage shifter techniques allow a emulsion track timing resolution of $<1 \mathrm{sec}$ with high reliability and high efficiency for large-scale and inaccessible emulsionic experiments [6]. We co-developed multi-stage shifter for the balloon experiment with Mitaka Kohki Co., Ltd. from November 2009. Figure 1 shows the multi-stage shifter for the balloon experiment. It has $145 \times$ $66 \times 10 \mathrm{~cm}^{3}$ dimensions, $75 \mathrm{~kg}$ weight, $25 \mathrm{~W}$ typical electric consumption. An aperture area is 
$3880 \mathrm{~cm}^{2}\left(38.8 \times 25.0 \mathrm{~cm}^{2} \times 4\right.$ emulsion film units per stage $)$. A number of stages is three with $1 \mathrm{~mm}$ gap between $1 \mathrm{st}$ stage and $2 \mathrm{nd}$ stage, and $2 \mathrm{~mm}$ gap between $2 \mathrm{nd}$ stage and $3 \mathrm{rd}$ stage. We performed major improvements by repeating various tests. We achieved a significant improvement for a low temperature performance. As a result, we obtained a performance for high speed operation (500 $\mu \mathrm{m} / \mathrm{sec}$ ) under low temperature (down to $-60{ }^{\circ} \mathrm{C}$ or below). It corresponds to $10 \mathrm{msec}$ timing resolution (with $5 \mu \mathrm{m}$ track connection accuracy). By improving the stage and emulsion film mounting on a stage, nearest distance between emulsion films on each stage can be improved to $0.7 \mathrm{~mm}$. Hereby, track connection accuracy can be improved to be less than $5 \mu \mathrm{m}$. As a result, msec order timing resolution can be achieved. Besides, a repeatability of return to origin under temperature variation was improved from $0.48 \mu \mathrm{m} /{ }^{\circ} \mathrm{C}$ to $0.11 \mu \mathrm{m} /{ }^{\circ} \mathrm{C}$ by improved origin sensor unit. Uniformity of gaps between stages across the whole area was improved from $0.11 \mathrm{~mm}$ to 0.05 $\mathrm{mm}$ standard deviation. The multi-stage shifter which has large aperture, high timing resolution and high quality can achieve Vela pulsar imaging. Moreover, phase resolving of the pulse emission with $89 \mathrm{msec}$ cycle from the Vela pulsar can also be challenged. More details of the development of the multi-stage shifter have been described in Ref. [7, 8].

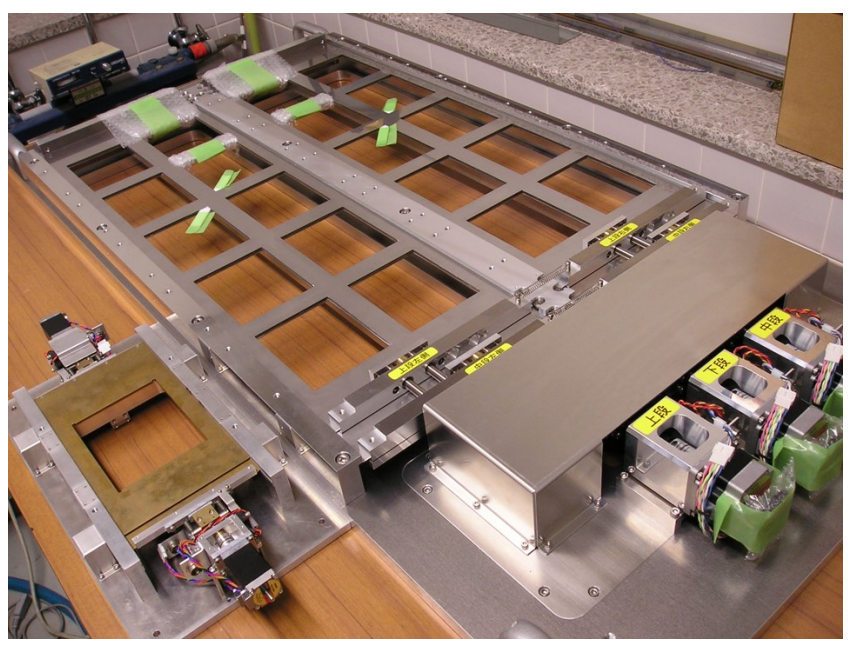

Figure 1: The flight model of multi-stage shifter.

We implemented vacuum packing of emulsion films in the balloon experiment. Vacuume packing can make robust emulsion film stack which can suppress slipping between emulsion films, make good contact between emulsion films and make good flatness. And it can shield from damp air due to airtight pack to avoid emulsion track fading. And it can achieve reliable recovery due to lighttight and watertight pack. To keep the vacuum pack, we implemented a pressure vessel. We developed ultra thin pressure vessel refered ATIC's pressure vessel. The pressure vessel can achieve $30 \mathrm{kPa}$ differential pressure with several $\mathrm{kPa}$ /day leak rate at room temperature to $-40{ }^{\circ} \mathrm{C}$. Upper material has $0.1 \mathrm{~g} / \mathrm{cm}^{2}$ to avoid gamma-ray absorption. More details of the pressure vessel are described in Ref. [9].

For star camera as an attitude monitor, to improve attitude determination efficiency and accuracy (especially around the line of sight), a hood length was modified, a CCD camera was selected with higher quantum efficiency (especially infrared band) and it is mounted with three directions 
for each 90 degree azimuth.

Emulsion film handling in Australia is essential for emulsion film processing before and after the experiment. The processes before the experiment have refreshing/resetting, drying and vacuum packing. Resetting/Refreshing is in order to initialize emulsion films before the experiment. This process keeps emulsion films under $25{ }^{\circ} \mathrm{C}$ temperature and $95 \%$ relative humidity for 24 hours. Drying is in order to suppress fading. This process keeps emulsion films under $25{ }^{\circ} \mathrm{C}$ temperature and $25 \%$ relative humidity for 24 hours. Vacuum packing is in order to be robust emulsion film stack, to avoid fading and to achieve reliable recovery. The process after the experiment has developing which is finalized process for the emulsion film. We considered and prepared the emulsion film hundling facility at Sydney University. We performed the start-up of the emulsion film hundling facility and the tests for refreshing/resetting, drying, vacuum packing and developing in 21st Feb - 15th March 2015.

\section{Emulsion chamber}

The aperture area has $3780 \mathrm{~cm}^{2}$ with $37.8 \times 25.0 \mathrm{~cm}^{2} \times 4$ units. The components have converter, timestamper, calorimeter, flatness compensation films and unit alignment films. The converter consists of the stack of 100 emulsion films (55\% occupied). The total thickness and radiation length $\left(X_{0}\right)$ of the converter were $32.0 \mathrm{~mm}$ and $0.53 X_{0}$, corresponding to a 0.34 conversion efficiency. The timestamper consists of the multi-stage shifter with three stages. The multi-stage shifter consisted of three stages, with 2 emulsion films (44\% occupied) on the top of the 1st stage, 1 emulsion films (44\% occupied and $40 \mu \mathrm{m}$ base) on the bottom of the 1st stage, 1 emulsion films (44\% occupied and $40 \mu \mathrm{m}$ base) on the top of the 2nd stage, 1 emulsion films (44\% occupied) on the bottom of the 2 nd stage and 3 emulsion films ( $44 \%$ occupied) on the top of the 3 rd stage. The calorimeter consists of a sandwich of 16 emulsion films (44\% occupied) and 15 SUS316L stainless steel plates. The total thickness and radiation length of the calorimeter were $19.3 \mathrm{~mm}$ and $0.90 X_{0}$, respectively.

\section{Emulsion film processing before the experiment}

We performed emulsion film processing before the experiment at the emulsion film handling facility in Sydney University. The processes have refreshing/resetting, drying and vacuum packing. We proccessed $509+$ backup emulsion films in 2nd - 17th April 2015. Packed emulsion films were shipped on 27 th April on the ground way kept below $10{ }^{\circ} \mathrm{C}$ with cooling medium exchange.

\section{Balloon experiment}

Final assembling was performed at BLS Alice Springs from the middle of April 2015. We checked on-board equipment (multi-stage shifter, star cameras, temperature meters, pressure meters, GPS systems, and a battery) and assembled them on the pressure vessel gondola. The emulsion films arrived from Sydney University on 1st May. After mounting emulsion films and rehearsing for the launch, we were ready for the flight on 5th May (Figure 2). Total weight of the gondola has $497 \mathrm{~kg}$ including JAXA's equipment without a ballast. After waiting for the wind condition, 
the balloon launch was performed on 12th May from the BLS. The balloon was launched at 6:33 ACST. Level flight at $37.2 \mathrm{~km}$ altitude started from 8:50 and the balloon went to the east direction. The observation was terminated at 20:00 and the gondola was released at 20:22. The gondola landed with a parachute at about $130 \mathrm{~km}$ north from Longreach at 20:55. The total flight duration in this experiment was 14.4 hours, with 11.5 hour level flight $(8: 50-20: 22)$ at $36.0-37.4 \mathrm{~km}$ altitude. Figure 3 and 4 show the flight path and altitude. The multi-stage shifter was operated from 6:35 with $10 \mu \mathrm{m} / \mathrm{sec}$ speed of 3rd stage. From 13:45, operation mode was changed with 500 $\mu \mathrm{m} / \mathrm{sec}$ speed of $3 \mathrm{rd}$ stage to cover Vela pulsar within 45 degree zenith with msec order timing resolution. The operation was terminated at 20:00. Figure 5 shows the repeatability of return to origin of 3rd and 2nd stage of the multi-stage shifter. The repeatability was obtained with $0.85 \mu \mathrm{m}$ RMS of 3rd stage in the $10 \mu \mathrm{m} / \mathrm{sec}$ operation, $0.14 \mu \mathrm{m}$ RMS of 3rd stage in the $500 \mu \mathrm{m} / \mathrm{sec}$ operation, and $0.33 \mu \mathrm{m}$ RMS of $2 \mathrm{nd}$ stage in the whole operation. The multi-stage shifter was successfully operated during the flight. Figure 6 shows a status of the pressure vessel during the flight. The pressure vessel kept with $270-240 \mathrm{hPa}$ during the flight. The gondola was successfully recovered from landing point to Longreach in the next day. Emulsion films were shipped from Longreach to Sydney on 18th May on the ground way kept almost below $10^{\circ} \mathrm{C}$ with cooling medium exchange.
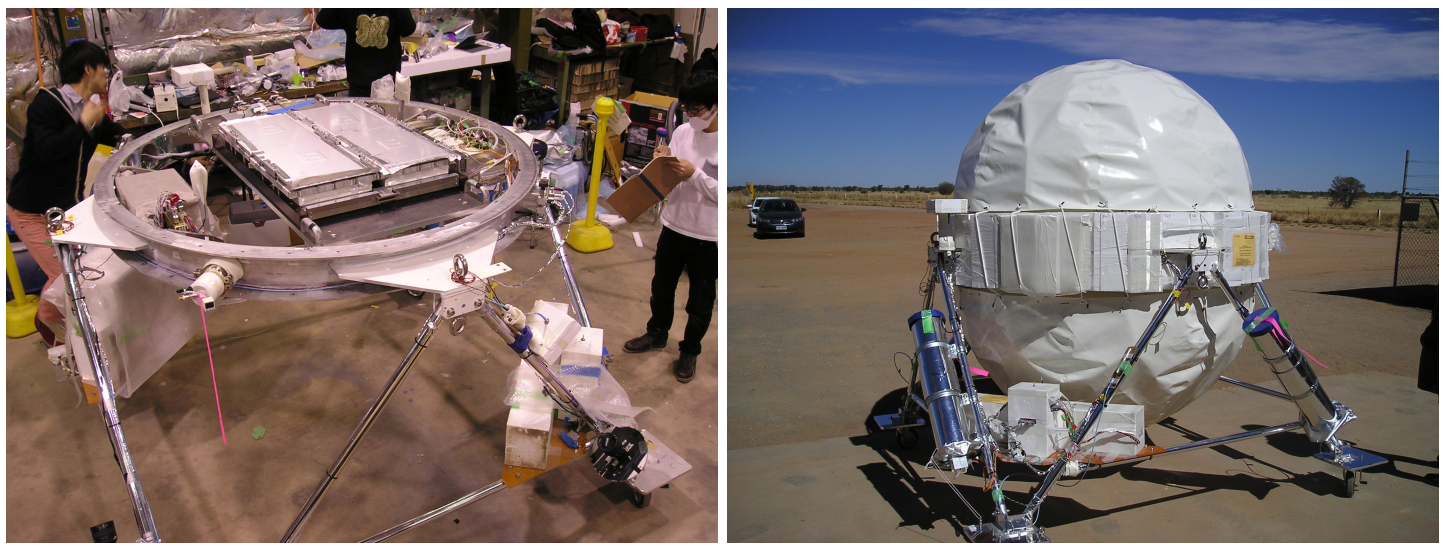

Figure 2: Two pictures show the gondola. (Left) Packed emulsion films were mounted on the multi-stage shifter on the ring of the pressure vessel. The star cameras without food can be seen on the truss, near the caster. (Right) The gondola after thermal shielding. The distance between casters has $2 \mathrm{~m}$.

\section{Emulsion film processing after the experiment}

We performed emulsion film processing after the experiment at the emulsion film handling facility in Sydney University. Emulsion films arrived at Sydney University from Longreach on 20th May. We started real development with 24 films/day from the next day and with 48 films/day from 23th May. The 48 films/day developing speed corresponds to 1100 OPERA films/day and 19 OPERA bricks/day developing speed in the silver amount base. We completed 509 emulsion film development on 1st June with negligible loss due to development error. And, for this mass production, we achieved a major revision of the capability of the developer with factor 2 . 
GRAINE project: An overview and status of the 2015 balloon-borne experiment with emulsion gamma-ray telescope Satoru Takahashi
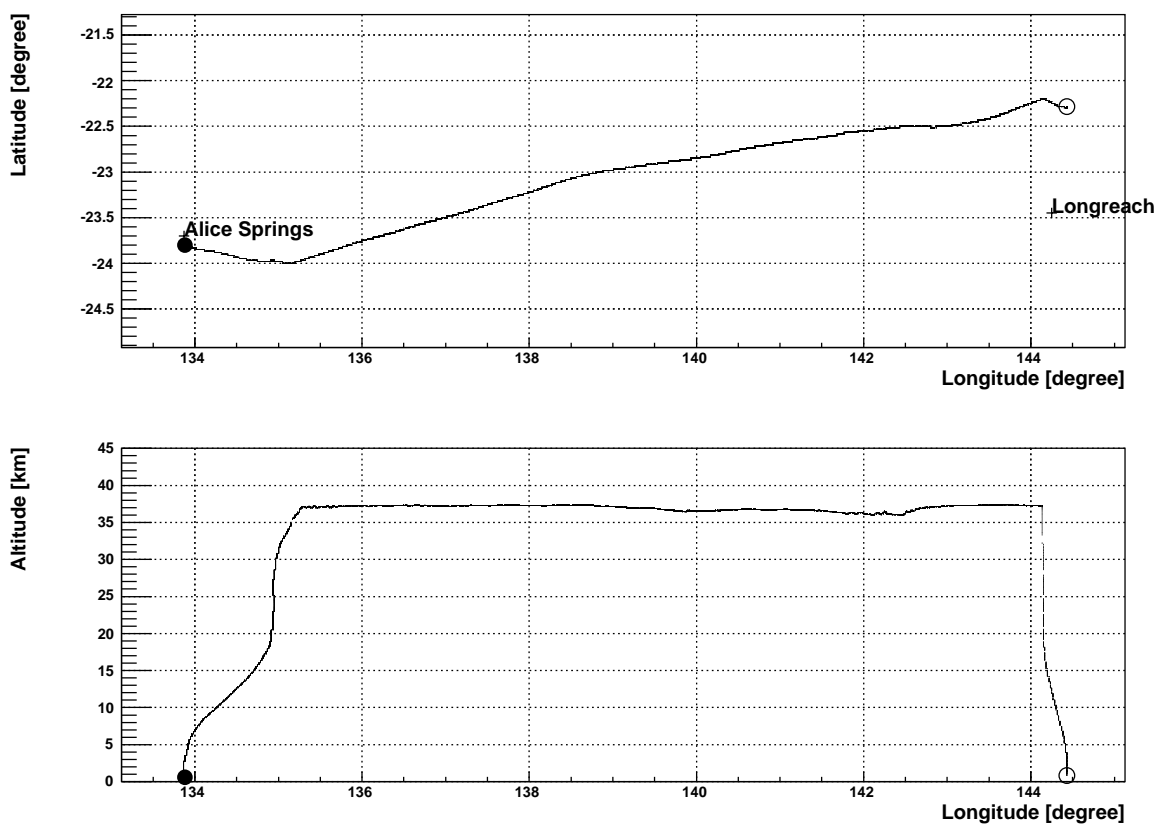

Figure 3: Top panel shows the flight path in longitude (horizontal axis) and latitude (vertical axis). The distance from Alice Springs to Longreach has $1059.2 \mathrm{~km}$. Bottom panel shows the flight path in longitude (horizontal axis) and altitude (vertical axis).

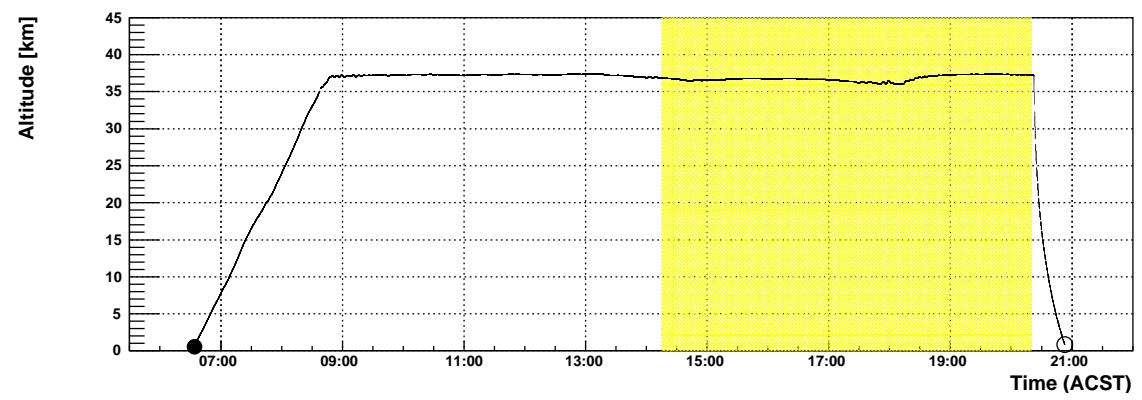

Figure 4: The altitude as a function of time. Hatched region shows the Vela pulsar within 45 degree zenith.

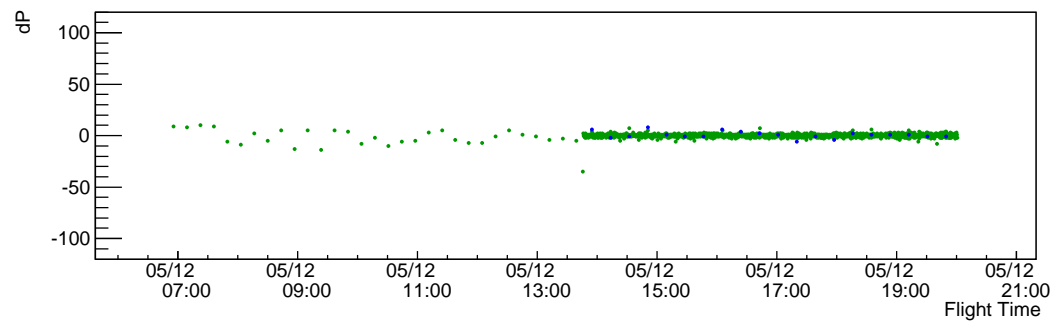

Figure 5: The repeatability of return to origin of 3 rd and 2 nd stage of the multi-stage shifter as a function of time. The vertical axis shows the repeatability in the difference of number of pulses between go and return ( $\mathrm{dP}) . \mathrm{dP}=1$ corresponds to $0.1 \mu \mathrm{m}$. The green plots and the blue plots show 3rd stage and 2nd stage, respectively. 


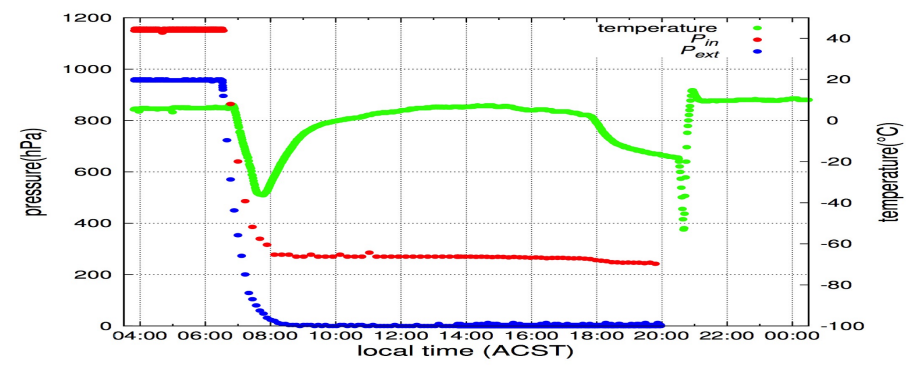

Figure 6: The status of the pressure vessel during the flight. The red plots show internal pressure of the vessel. The blue plots show external pressure. The red plots show a temperature of the bottom shell of the vessel inside of the thermal shield shell.

\section{Conclusion}

We completed the balloon experiment. The emulsion films are scanned across the whole area with Hyper Track Selector developed by F-lab., Nagoya University. We are starting the flight data analysis. Current status of the flight data analysis is described in Ref. [10].

\section{Acknowledgments}

The balloon-borne experiment was conducted by Scientific Ballooning (DAIKIKYU) Research and Operation Group, ISAS, JAXA. The final preparation and launch were performed at Alice Springs, Balloon Launching Station managed by R. Sood et al., University of New South Wales. Emulsion film transfer in Australia and so on were adviced and supported by D. Sullivan. Emulsion film processing before and after the experiment were supported by A. Bakich, K. Varvell, D. Beech et al., University of Sydney. Emulsion film pouring and storage were supported by K. Hoshino and K. Nakazawa et al., Gifu University. This work was supported by JSPS KAKENHI (Grant Numbers 20244031, 26105510, 26247039, and 26800138) and a Grant-in-Aid for JSPS Fellows.

\section{References}

[1] S. Aoki et al., arXiv:1202.2529.

[2] S. Takahashi et al.,Proc. 33rd Int. Cosmic Ray Conference, 228 (2013).

[3] S. Takahashi et al., Prog. Theor. Exp. Phys. 043H01 (2015).

[4] H. Rokujo et al., Nucl. Instrum. Meth. A 701, 127 (2013).

[5] H. Rokujo et al., Proc. 34rd Int. Cosmic Ray Conference, 1009 (2015).

[6] S. Takahashi et al., Nucl. Instrum. Meth. A 620, 192 (2010).

[7] S. Takahashi et al., Ionizing radiation, Vol.40, No.3 (2014) [in Japanese].

[8] F. Mizutani et al., Proc. Balloon Symp., ISAS/JAXA, isas14-sbs-005 (2014) [in Japanese].

[9] H. Rokujo et al., Proc. 34rd Int. Cosmic Ray Conference, 1003 (2015).

[10] K. Ozaki et al., Proc. 34rd Int. Cosmic Ray Conference, 869 (2015). 